\title{
Subgingival microbiota of dogs with healthy gingiva or early periodontal disease from different geographical locations
}

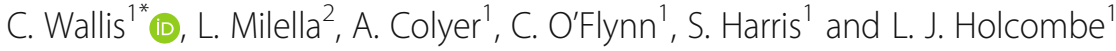

\begin{abstract}
Background: Periodontal disease is the most common oral disease of dogs worldwide and results from a complex interplay between plaque bacteria, the host and environmental factors. Recent studies have enhanced our understanding of the associations between the plaque microbiota and canine periodontal disease. These studies, however, were limited in their geographical reach. Thus associations between the canine oral microbiota and geographical location were investigated by determining the composition of subgingival plaque samples from 587 dogs residing in the United Kingdom (UK), United States of America (USA), China and Thailand using 454pyrosequencing.

Results: After quality filtering 6,944,757 sequence reads were obtained and clustering of these at $\geq 98 \%$ sequence resulted in 280 operational taxonomic units (OTUs) following exclusion of rare OTUs (present at $<0.05 \%$ in all four countries). The subgingival plaque from dog populations located in the UK, USA, China and Thailand had a similar composition although the abundance of certain taxa varied significantly among geographical locations. Exploration of the effect of clinical status and age revealed a marked similarity among the bacteria associated with increased age and those associated with gingivitis: Young dogs and those with no gingivitis were dominated by taxa from the phyla Bacteroidetes and Proteobacteria whereas older dogs and those with moderate gingivitis were dominated by members of the Firmicutes. The plaque microbiota of small breed dogs was found to significantly differ to medium and large breeds and was dominated by species belonging to the Firmicutes.

Conclusions: The bacterial associations with health, gingivitis and periodontitis were conserved across dogs from the UK, USA, China and Thailand. These bacterial signatures of periodontal health and disease have potential as biomarkers for disease detection.
\end{abstract}

Keywords: Microbiota, Plaque, Periodontal disease, Gingivitis, Canine, Geography

\footnotetext{
*Correspondence: Corrin.wallis@effem.com

'WALTHAM Petcare Science Institute, Mars Petcare UK, Melton Mowbray, Leicestershire, UK

Full list of author information is available at the end of the article
}

(c) The Author(s). 2021 Open Access This article is licensed under a Creative Commons Attribution 4.0 International License, which permits use, sharing, adaptation, distribution and reproduction in any medium or format, as long as you give appropriate credit to the original author(s) and the source, provide a link to the Creative Commons licence, and indicate if changes were made. The images or other third party material in this article are included in the article's Creative Commons licence, unless indicated otherwise in a credit line to the material. If material is not included in the article's Creative Commons licence and your intended use is not permitted by statutory regulation or exceeds the permitted use, you will need to obtain permission directly from the copyright holder. To view a copy of this licence, visit http://creativecommons.org/licenses/by/4.0/ The Creative Commons Public Domain Dedication waiver (http://creativecommons.org/publicdomain/zero/1.0/) applies to the data made available in this article, unless otherwise stated in a credit line to the data. 


\section{Background}

Periodontal disease is the most common oral disease of dogs worldwide [1-3]. Clinically the first sign is red and inflamed gums, gingivitis, which can progress into periodontitis where the ligaments and bones that support the tooth are progressively destroyed [4]. The initial stages of the disease can be managed with early identification and intervention, however, without effective treatment the chronic inflammation and tissue damage is likely to cause significant pain and tooth loss in the latter stages of the disease.

The disease results from a complex interplay between plaque bacteria, the host and environmental factors. Early culture-based studies to understand the role of bacteria in periodontal disease highlighted that canine bacterial species were different to those of humans [5-7]. This finding was supported by a subsequent molecular study which identified 353 taxa of which $80 \%$ were novel and only $16.4 \%$ were shared with the human oral microbiota [8]. Recently, advances in sequencing technologies coupled with new bioinformatics developments have enabled studies that have significantly enhanced our understanding of the canine bacterial species associated with health and periodontal disease [9-11].

Although the importance of the oral microbiota for canine health and disease is increasingly recognised, to the best of our knowledge there have been no studies to explore the variability in the composition of the canine oral microbiota across different geographies. Several studies of human subjects have shown that the mean proportions of plaque-associated species differ by country [12-14] and that there are oral microbial signatures capable of differentiating between ethnicities and between populations living in different geographical locations [15-19]. Furthermore, studies of isolated communities of people have resulted in identification of a number of novel taxa not previously described, resulting in a distinctive composition of the oral microbiota [20, 21]. In contrast, other investigators have concluded that the oral microbiota was not associated with the geographic regions studied [22, 23].

The aim of this study was to investigate associations between the canine oral microbiota and geographical location in a cross-sectional study of dogs from the United Kingdom (UK), United States of America (USA), China and Thailand. The influence of periodontal health status, age and breed size were also taken into consideration as it was not possible to control for the cohorts of dogs presenting at the veterinary clinics. The hypothesis was that bacterial associations with periodontal health and disease will be conserved across individuals irrespective of the country in which they reside.

\section{Results}

Study cohort

Subgingival plaque samples from 587 dogs were included in the study. Breed size and age are potential risk factors for periodontitis and therefore sample associated metadata were also obtained (Table 1). Due to missing data, there were ten dogs of unknown age from China, four dogs of unknown breed size and one dog of unknown sex from the UK. The cohort of dogs from Thailand had a significantly lower age than dogs from the other three geographical locations $(p<0.001)$. Chisquared analysis showed that there was a significant difference in the distribution of breed size across the dogs sampled in each of the four geographical locations $(p<0.001)$. There was no significant difference in the ratio of males to females $(p=0.971)$.

The sample collection procedure differed between the UK and the other three geographical locations (see methods) and therefore clinical status was determined on a whole mouth and on a teeth sampled basis. There were significant differences in the clinical status of dogs across the four locations as shown in Table 2. For example, the dogs from China had a higher average gingivitis score and a lower percentage of healthy teeth (G0) than the other geographical locations. Dogs from Thailand had a higher percentage of teeth with periodontitis and the percentage of missing teeth was lower in the UK dogs compared to dogs from China, Thailand and USA.

Further exploration of the correlations between the clinical status of the whole mouth compared to the sampled teeth showed that the USA, Thailand and China were almost identical due to nearly all the teeth in the mouth being sampled (supplementary figure 1). The exceptions were 33 dogs with >PD2 (periodontal disease stage 2) teeth in the mouth and two dogs with PD2 teeth in a "healthy mouth" that were not included in the sample. For the UK samples there was correlation with respect to health and gingivitis although there was less correlation than the other three geographical locations (supplementary figure 1A \& 1B). However, with respect to periodontitis the correlation was poor, defined by the divergence as the number of periodontitis teeth sampled increased (supplementary figure $1 C$ ). This occurs due to the fact that in most instances the UK dogs only had 68 periodontitis teeth sampled and no other teeth in the mouth included.

\section{Sequence quality}

Analysis of the 587 subgingival plaque samples by 454pyrosequencing of the 3 ' end of the V1-V3 region of the $16 \mathrm{~S}$ rDNA gene resulted in the generation of $10,537,181$ sequence reads that passed the sequencing providers initial sequence quality filter. After AmpliconNoise filtering 
Table 1 Summary of metadata for each of the four locations

\begin{tabular}{|c|c|c|c|c|}
\hline & UK & USA & China & Thailand \\
\hline \multicolumn{5}{|l|}{ Age (years) } \\
\hline Average & 5.78 & 5.61 & 5.02 & 2.67 \\
\hline$(95 \% \mathrm{Cl})$ & $(5.26,6.3)^{b}$ & $\begin{array}{l}(4.93 \\
6.29)^{b}\end{array}$ & $\begin{array}{l}(4.17 \\
5.87)^{b}\end{array}$ & $\begin{array}{l}(2.16 \\
3.18)^{a}\end{array}$ \\
\hline Range & 1.5 to 15 & 1 to 14.5 & 1 to 14 & 0.8 to 14 \\
\hline \multicolumn{5}{|l|}{ Breed size } \\
\hline Small $(<10 \mathrm{~kg})$ & $36(17.6 \%)$ & $56(46.3 \%)$ & 57 (42.5\%) & $22(17.7 \%)$ \\
\hline $\begin{array}{l}\text { Medium (10-25 } \\
\mathrm{kg})\end{array}$ & 76 (36.9\%) & $24(19.8 \%)$ & $43(32.1 \%)$ & 81 (65.3\%) \\
\hline Large (>25 kg) & $93(45.1 \%)$ & 41 (33.9\%) & $33(24.8 \%)$ & 21 (16.9\%) \\
\hline \multicolumn{5}{|l|}{ Sex } \\
\hline Female & $94(45.2 \%)$ & $54(44.6 \%)$ & 55 (45.1\%) & $59(47.6 \%)$ \\
\hline Male & $\begin{array}{l}114 \\
(54.5 \%)\end{array}$ & $67(54.5 \%)$ & $67(54.5 \%)$ & 65 (52.4\%) \\
\hline
\end{tabular}

${ }^{\mathrm{a}, \mathrm{b}}$ Significance with Tukey HSD homogenous groups at $5 \%$ within model

$6,944,757$ sequence reads remained. The number of sequences per sample ranged from 2027 to 30,050. The mean number of sequences per sample was 13,868 with $95 \%$ confidence interval $(12,912,14,825), 12,016$ (11,321, 12,710), 8807 (8110, 9503) and 11,460 (10,944, 11,976) for UK, USA, China and Thailand respectively. Generalised linear model (GLM) analysis showed that the UK sequence depth was more variable than the other countries and had a significantly higher sequence depth on average than all other geographical locations $(p<0.001)$. China had a lower sequence depth on average than all other geographical locations $(p<0.001)$.

\section{Bacterial composition of canine plaque}

A total of 13,530 OTUs were identified by clustering at $\geq 98 \%$ sequence identity and of these 13,250 were rare; 7819 OTUs had less than two counts across all geographical locations and 5431 OTUs had no geographical location with $>0.05 \%$ sequence reads. The percentage of these rare sequences did not significantly differ between geographical locations with mean values of $3.64 \%$ (3.22, 4.11), $3.13 \%$ (2.60, 3.77), $3.56(2.92,4.31)$ and $3.09(2.55,3.73)$ in
UK, USA, China and Thailand respectively $(p>0.05)$. After exclusion of the rare OTUs a total of 280 OTUs remained.

Of the 280 OTUs, 254 (91\%) shared $\geq 98 \%$ sequence identity and $\geq 98 \%$ coverage to sequences within the Silva database. Of these 140 (50\%) mapped to sequences from a previous study of the canine oral microbiota (Dewhirst et al., 2012; Genbank accession numbers JN713151-JN713566 \& KF030193-KF030235). Thirty (11\%) and $71(25 \%)$ mapped to sequences annotated to the species and genus level respectively and the remaining $14(5 \%)$ had only been annotated to the family, order or phylum level. For the remaining 25 (9\%) OTUs the percentage identity or coverage was less than $98 \%$ but all had $\geq 95 \%$ sequence identity to sequences within the Silva database and $\geq 86.6 \%$ coverage.

Overall eleven phyla were identified and the proportions of each were similar across all four geographical locations (Fig. 1). Firmicutes were the most abundant on average across all samples (29.4\%) followed by Actinobacteria (22.5\%), Bacteroidetes (19.8\%), Proteobacteria (14.9\%), Fusobacteria (3.6\%), Spirochaetes (2.5\%), TM7 (1.5\%), Chloroflexi (0.76\%), Synergistetes (0.63\%), Chlorobi $(0.63 \%)$ and SR1 (0.36\%).

At $\geq 98 \%$ sequence identity, which approximates to species level, Porphyromonas cangingivalis was the most dominant taxa with an average abundance of $5.7 \%$ across all samples. A further 23 OTUs had average relative abundances $>1 \%$ and together accounted for $39.4 \%$ of sequence reads (Table 3). Of the remaining OTUs, 128 were present with average proportions ranging from 0.1 to $1 \%$ and these accounted for $45.7 \%$ of the sequence reads, and 128 OTUs had average proportions $<0.1 \%$ and together these accounted for $6.8 \%$ of the sequence reads.

\section{Composition of subgingival plaque}

The composition of subgingival plaque was investigated and clearly showed evidence of a core microbiota in canine subgingival plaque based on presence of OTUs (presence was defined as $>0.05 \%$ ); that is a set of

Table 2 Summary of clinical status of dogs across four locations

\begin{tabular}{lllll}
\hline Model & \multicolumn{2}{l}{ Location } & & \\
\cline { 2 - 5 } & UK & USA & China & Thailand \\
\hline Average gingivitis score in mouth & $1.2(1.05,1.35)^{\mathrm{a}}$ & $1.13(0.95,1.32)^{\mathrm{a}}$ & $1.41(1.29,1.53)^{\mathrm{b}}$ & $1.06(0.92,1.2)^{\mathrm{a}}$ \\
Average gingivitis score sampled teeth & $1.35(1.18,1.51)^{\mathrm{bc}}$ & $1.13(0.95,1.31)^{\mathrm{ab}}$ & $1.41(1.29,1.52)^{\mathrm{c}}$ & $1.06(0.92,1.19)^{\mathrm{a}}$ \\
\% Healthy teeth in mouth & $35.01(28.82,41.75)^{\mathrm{a}}$ & $36.74(28.55,45.78)^{\mathrm{a}}$ & $11.34(6.9,18.07)^{\mathrm{b}}$ & $37.73(29.55,46.67)^{\mathrm{a}}$ \\
\% Healthy sampled teeth & $33.87(25.44,43.47)^{\mathrm{a}}$ & $36.9(29.65,44.8)^{\mathrm{a}}$ & $11.38(7.39,17.14)^{\mathrm{b}}$ & $37.86(30.63,45.67)^{\mathrm{a}}$ \\
\% periodontitis teeth in mouth & $7.81(6,10.1)^{\mathrm{b}}$ & $6.19(4.17,9.1)^{\mathrm{b}}$ & $6.11(4.17,8.38)^{\mathrm{b}}$ & $13.29(10.26,17.03)^{\mathrm{a}}$ \\
\% periodontitis teeth sampled & $10.99(7.75,15.35)^{\mathrm{a}}$ & $5.78(3.86,8.58)^{\mathrm{b}}$ & $5.74(3.89,8.38)^{\mathrm{b}}$ & $13.03(10.08,16.69)^{\mathrm{a}}$ \\
\% missing teeth in mouth & $2.24(1.5,3.35)^{\mathrm{a}}$ & $3.66(2.42,5.5)^{\mathrm{ab}}$ & $4.48(3.14,6.35)^{\mathrm{b}}$ & $3.76(2.52,5.6)^{\mathrm{bb}}$ \\
\hline
\end{tabular}

$\bar{a}, b, c$ Significance with Tukey homogeneous groups at $5 \%$ within model 


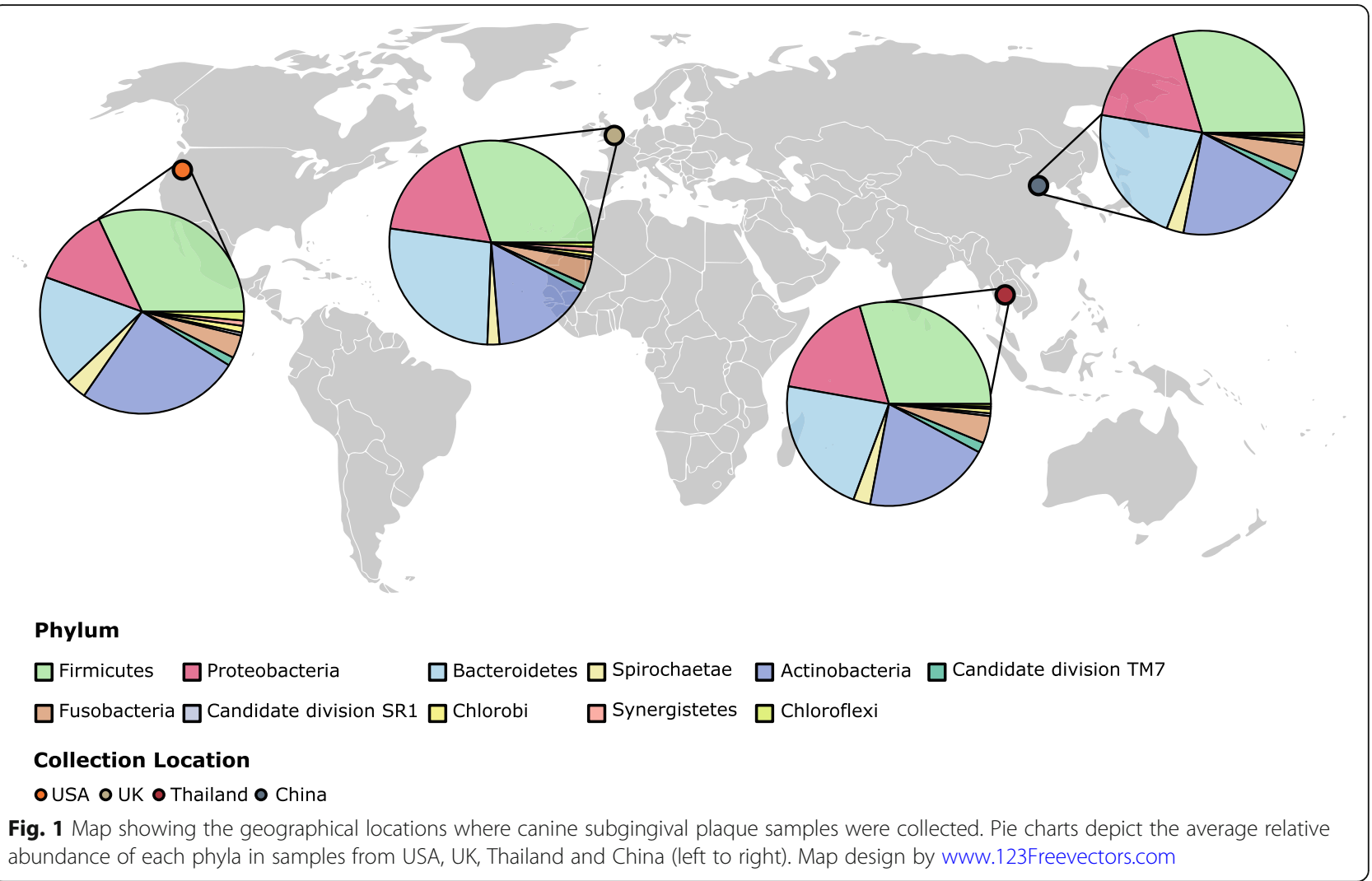

common OTUs across all dogs and geographical locations (supplementary figure 2). The OTUs formed three main clusters of similarly present OTUs which together accounted for $46.6 \%$ of the total sequences (supplementary figure 2). Cluster A contains 10 OTUs (3.6\% of the total number of OTUs), cluster B contains 16 OTUs (5.7\%) and cluster $\mathrm{C}$ comprises 50 OTUs (17.9\%).

For cluster A, the percentage of samples in which the OTU was present ranged from 4.9 to $84.7 \%$ with all but 2 OTUs present in more than $65 \%$ of samples. The OTUs were distributed across two phyla; Proteobacteria (6 OTUs) and Bacteroidetes (4 OTUs). The most abundant taxa were Neisseria shayeganii (1.3\%) and novel, as yet unclassified, species from the genera Bergeyella (2.0\%) and Moraxella (3.0\%). The percentage of samples positive for the OTUs in cluster B ranged from 17 to $94.5 \%$ with the majority occuring in more than $78 \%$ of samples. The most represented phyla were Firmicutes (6 OTUs), Bacteroidetes (3 OTUs) and Actinobacteria (4 OTUs). Five OTUs were present in $>90 \%$ of the samples; two species belonged to the genus Actinomyces (Actinomyces sp. COT-252, Actinomyces sp. COT-083) and the other species were a novel unclassified species belonging to the genus Fusobacterium, TM7 phylum sp. COT-305 and Peptostreptococcaceae bacterium COT-004. A further four OTUs were present in greater than $80 \%$ of samples and had an average relative abundance of $>1 \%$; Erysipelotrichaceae bacterium COT-
311, Filifactor villosus and, novel species from the genera Euzebya and Johnsonella. With respect to cluster C the percentage of samples in which the OTUs were present ranged from 3.1 to $91.3 \%$ with the majority (70\%) present in more than $50 \%$ of samples. One OTU, a novel unclassified species belonging to the genus Fusobacterium was present in $>90 \%$ of samples. Seven OTUs within this cluster were present at $>1 \%$ and present in at least $50 \%$ of samples: Porphyromonas canoris, Clostridiales bacterium COT-028, Porphyromonas gulae, Granulicatella sp. COT095 and novel species from the genera Corynebacterium and Euzebya.

There were only nine OTUs that were not present in all four countries. Five OTUs were absent in plaque collected from dogs in Thailand but present in dogs from the other three geographical locations: Actinomyces sp. COT-412 was present at $>0.07 \%$ in USA, UK and China, a further three OTUs (novel species from the genera Murdochiella and Actinomyces and a novel species from the family Christensenellaceae) were present at $<0.03 \%$ in USA and UK but $>0.05 \%$ in China, Escherichia-Shigella was prevalent in the UK dogs (> $0.067 \%$ ) but present at $<0.0004 \%$ in China and the USA. Streptococcus orisasini (prevalence 0.044\%) was only present in dogs from the UK and completely absent from dogs in the USA, China and Thailand and another novel Streptococcus species was present at $0.056 \%$ in the 
Table 3 Operational taxonomic units with an average proportion $>1 \%$ in canine subgingival plaque from dogs with healthy gingiva, gingivitis and mild periodontitis (all locations)

\begin{tabular}{ll}
\hline Taxa & $\begin{array}{l}\text { Proportion of total } \\
\text { sequence reads }\end{array}$ \\
\hline Porphyromonas cangingivalis & $5.67 \%$ \\
Novel Actinomyces & $5.08 \%$ \\
Novel Moraxella & $2.95 \%$ \\
Peptostreptococcaceae bacterium COT-077 & $2.55 \%$ \\
Actinomyces sp. COT-083 & $2.51 \%$ \\
Novel Bergeyella & $2.00 \%$ \\
Clostridiales bacterium COT-028 & $1.86 \%$ \\
Actinomyces sp. COT-252 & $1.69 \%$ \\
Novel Fusobacterium & $1.59 \%$ \\
Filifactor villosus & $1.59 \%$ \\
Novel Porphyromonas & $1.52 \%$ \\
Novel Peptostreptococcus & $1.46 \%$ \\
Novel Euzebya & $1.39 \%$ \\
Novel Corynebacterium & $1.35 \%$ \\
Peptostreptococcaceae bacterium COT-004 & $1.32 \%$ \\
Neisseria shayeganii & $1.30 \%$ \\
Novel Porphyromonas & $1.30 \%$ \\
Erysipelotrichaceae bacterium COT-311 & $1.29 \%$ \\
Granulicatella sp. COT-095 & $1.19 \%$ \\
Norphyromonas gulae & $1.14 \%$ \\
Novel Cornsonella & $1.11 \%$ \\
\hline & $1.11 \%$ \\
\hline
\end{tabular}

UK dogs and present at $<0.02 \%$ in China and Thailand but absent from the USA dogs. Another novel Streptococcus species was present at $>0.1 \%$ in USA dogs but was absent from dogs located in Thailand and China and present at $<0.006 \%$ in UK dogs. Finally, a novel Actinomyces species was present at high abundance in dogs from Thailand $(>0.06 \%)$ and UK dogs and present at $<0.015 \%$ in dogs from China but absent from USA.

\section{Effect of geographical location, clinical status, age and} dog breed size on the plaque microbiota

An exploratory principal components analysis (PCA) showed no discrete clustering of plaque microbiota by geographical location; the variance was possibly larger in the UK, although the number of dogs sampled was greatest in this geographical location (Fig. 2a). No discrete clustering was seen by breed size (Fig. 2b), sex or neuter status (data not shown). However, progression was evident in component one with increasing age suggesting that the variance in the OTUs changes with advancing age (Fig. 2c). Likewise, clinical status showed progression in component one suggesting the variance changes with percentage healthy teeth and average gingivitis (Fig. 2d and e). Progression was also evident with respect to the number of teeth with periodontitis although the pattern was not as clear (Fig. 2f). This indicates that the composition of the plaque microbiota is influenced by the extent of gingivitis and periodontitis. Similar patterns were evident for both the mouth and sampled teeth with respect to percentage of healthy teeth, average gingivitis and percentage of periodontitis teeth suggesting that clinical status of the mouth and the teeth sampled are highly correlated (data not shown). Therefore, only the sampled teeth data were investigated further to identify bacterial associations with geographical location, age, clinical status and breed size.

Of the 280 OTUs analysed by GLM, 237 had significant effects and of these 14 had significant geographical location interactions and 28 had interactions with other covariates (gingivitis, age, breed size, percentage healthy teeth and percentage of periodontitis teeth).

\section{Effect of geographical location}

Of the 14 significant interactions with geographical location, only six OTUs had an interaction between geographical location and clinical status and none with percentage healthy or periodontitis teeth, suggesting that bacterial associations with health or disease did not change by geographical location. There were also six interactions between geographical location and age and two with breed size (Supplementary table 1). Of the models without significant geographical location interactions, 152 OTUs had significant geographical location effects indicating that the relative abundance of bacteria significantly differed by geographical location (Supplementary table 2). Of the 912 pairwise comparisons, 355 were significant and the majority of these were due to differences between the UK and the other three countries $(24.5 \%$ of OTUs significantly different between the UK and China, 20\% significantly different between the UK and Thailand and 19.4\% significantly different between the UK and USA). The next most different were Thailand and China (14.4\% of pairwise comparisons significant), followed by the USA and China (11.3\%) and then USA and Thailand (10.4\%).

The UK was characterised by significantly higher estimated mean proportions of OTUs from the phyla Bacteroidetes and Proteobacteria and significantly lower estimated proportions of OTUs from the phyla Actinobacteria compared to the USA, Thailand and China (Supplementary table 2, Fig. 3). A number of OTUs belonging to the Firmicutes were also significantly less abundant in the UK compared to the other countries 


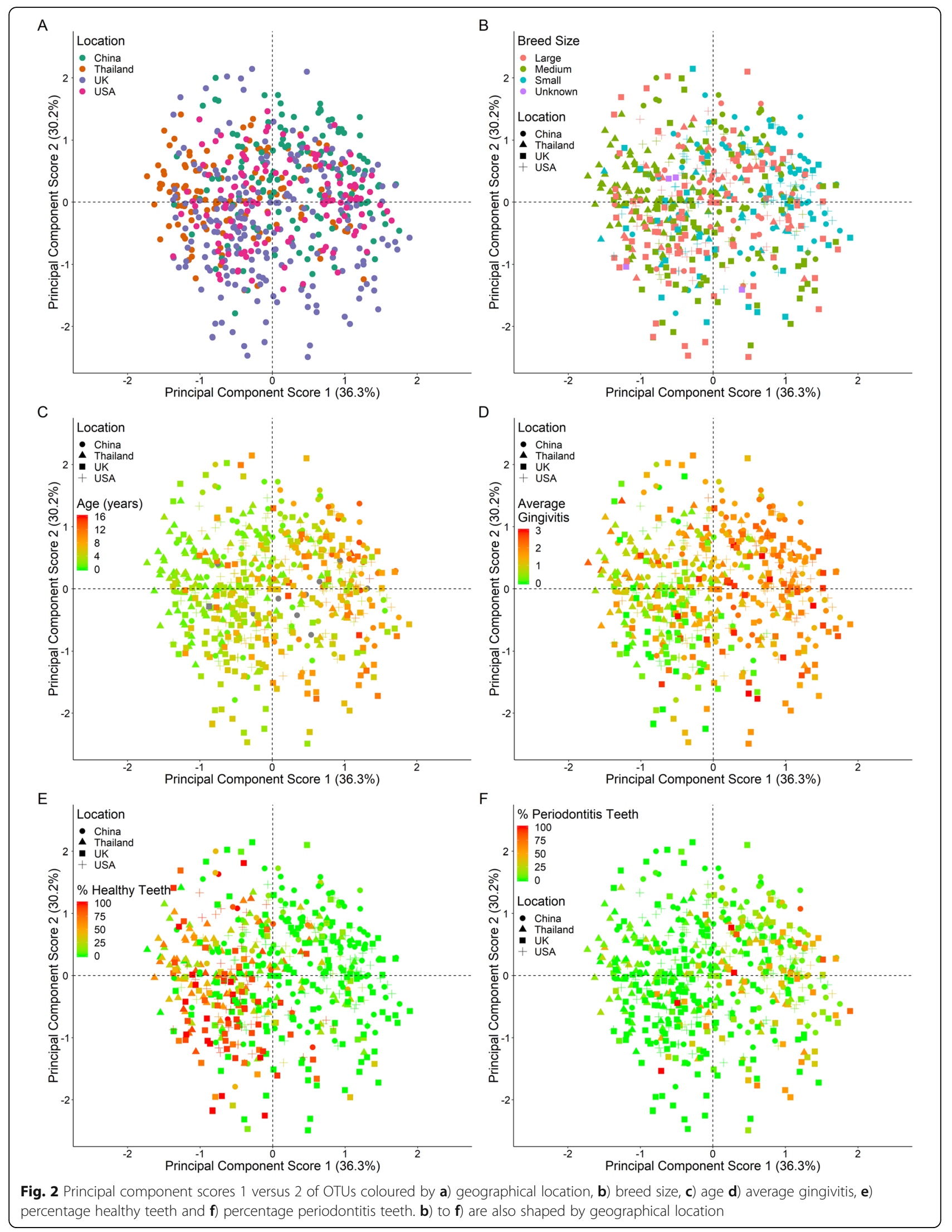



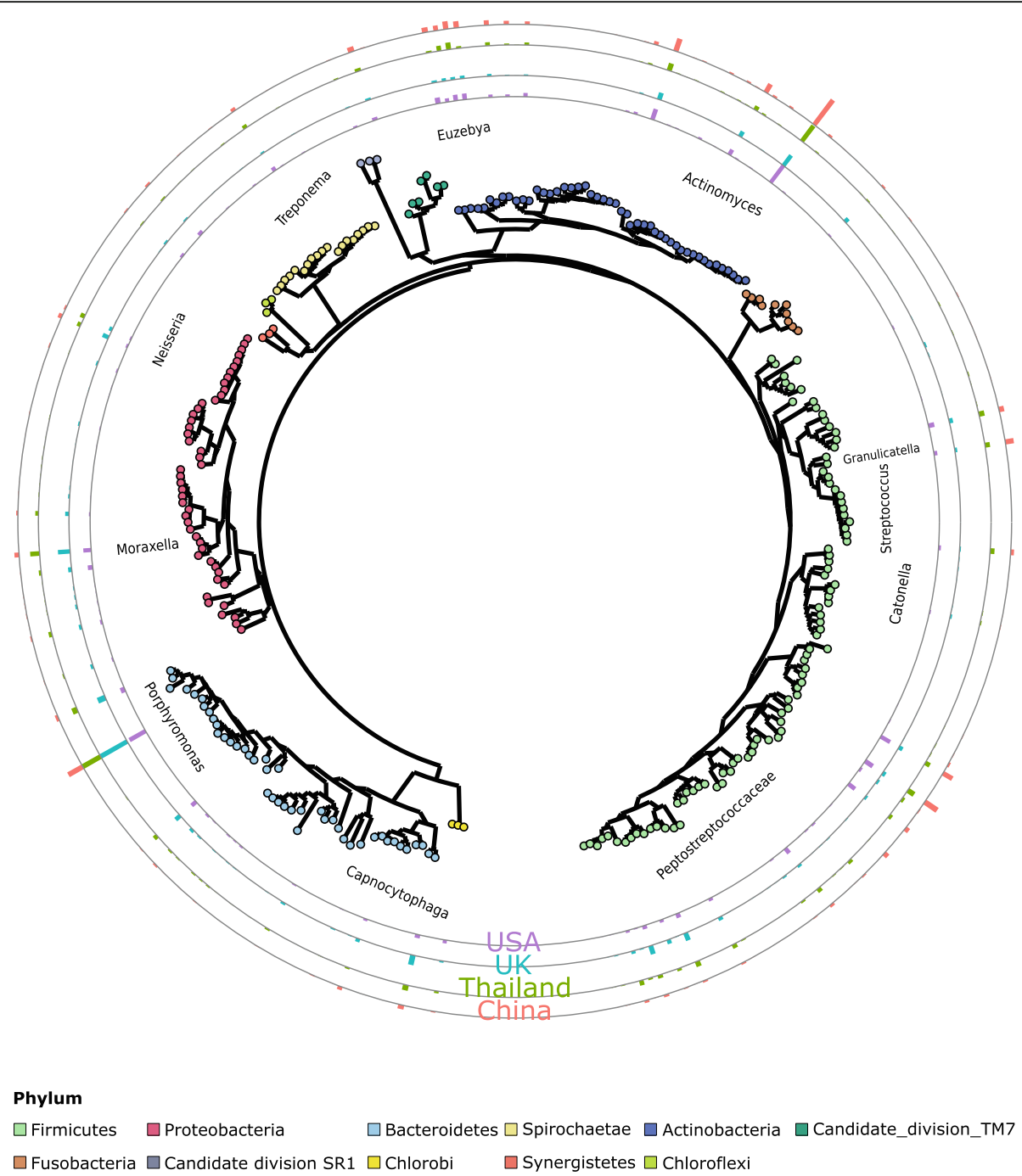

Fig. 3 Phylogenetic tree of representative sequences for 280 OTUs. Nodes are coloured by best hit phylum classification, notable clades are highlighted in grey. Ring annotations represent mean relative abundances for the four countries surveyed; from out to in, China in red, Thailand in green, UK in blue and USA in purple. The 153 OTUs with geographic significant effects have coloured ring annotations, whereas nonsignificant OTUs ring annotations are grey

but other OTUs belonging to this phyla had significantly higher estimated abundances. Within the phylum Bacteroidetes the most abundant OTUs $(>0.3 \%)$ showing the biggest differences between the UK and the other three countries (odds ratio $>2$ ) included two novel species from the genera Bergeyella and Porphyromonas. Within the phylum Proteobacteria the most notable differences were with respect to the species Cardiobacterium sp. COT-177, Desulfomicrobium orale and Pasteurella dagmatis which were all significantly more abundant in the UK compared to all other countries. With respect to the Firmicutes, two novel species from the genera Peptococcus and Peptostreptococcus and members of the family Peptostreptococcaceae were significantly more abundant in the UK compared to Thailand, China and USA. Members of the Actinobacteria that had a significantly lower estimated proportion in the UK compared to the other countries included two novel species from the genus Euzebya and two species from the genus Actinomyces (A. weissii and a novel species). With respect to the Firmicutes that had a significantly lower estimated abundance in the UK compared to other countries none were common to all three countries. One other phyla worthy of mention is Spirochaetes where one species, Treponema denticola, was significantly lower in the UK population compared to the other three countries.

Dogs from the USA were characterised by having higher estimated mean proportions of OTUs from the phylum Firmicutes compared to China and Thailand (Supplementary 
Table 2). Of the most abundant OTUs (>0.3\%) with the biggest difference between countries (odds ratio $>2$ ) only one species of Firmicutes, Helcococcus sp. COT-069 had a significantly higher proportion in the USA compared to Thailand and China. Plaque collected from dogs in the USA also had higher estimated proportions of Actinobacteria and Proteobacteria compared to Thailand and higher estimated proportions of OTUs from the phyla Bacteroidetes compared to China. The USA had lower estimated proportions of OTUs from the phyla Proteobacteria compared to Thailand and lower estimated proportions of OTUs from the phyla Actinobacteria than China.

In terms of the OTUs that differed in their mean relative abundance between Thailand and China, the phyla Firmicutes, Proteobacteria and Bacteroidetes had higher estimated proportion in Thailand compared to China. The most abundant $(>0.3 \%)$ Firmicutes with the biggest difference between the two countries (odds ratio $>2$ ) were Frigovirgula sp. COT-007, Tissierella sp. COT-029, a novel species from each of the genera Catonella and Parvimonas, a novel species from the family Erysipelotrichaceae and four species from the family Peptostreptococcaceae. With respect to the Proteobacteria, Campylobacter sp. COT-011 and two species form the genus Moraxella (COT-018 and a novel species) were the most notable. In terms of the phylum Bacteroidetes, Bacteroides sp. COT-040, Porphyromonas gulae and Prevotella sp. COT-226 had higher estimated proportions in Thailand compared to China. A number of OTUs from the phylum Actinobacteria were significantly less abundant in Thailand compared to China, for example, Actinobacteria bacterium COT-376, Actinomyces sp. COT-252, Propionibacterium sp. COT-296, a novel species from the genus Actinomyces and two novel species from the genus Corynebacterium.

\section{Associations with health, gingivitis and periodontitis} Of the 15 interactions with average gingivitis, eight were with percentage of teeth with periodontitis and three with the percentage of healthy teeth (Supplementary Table 1). When none of the sampled teeth had periodontitis there were three OTUs that had significantly higher estimated mean proportions when moderate gingivitis compared to no gingivitis and all belonged to the phylum Firmicutes (Peptostreptococcaceae bacterium COT-068, a novel species belonging to the genus Peptostreptococcus and Clostridiales bacterium COT-027). When all of the sampled teeth had periodontitis there were five OTUs that had significantly higher estimated proportions with moderate gingivitis compared to no gingivitis and these belonged to the phyla Proteobacteria (Campylobacter sp. COT-011), Bacteroidetes (Bacteroides sp. COT-040 and a novel species from the genus Alloprevotella) and Firmicutes (Frigovirgula sp. COT-007 and a novel species from the genus Fusobacter). With respect to the interaction between gingivitis and percentage healthy teeth, there were two OTUs, belonging to the phyla Actinobacteria (a novel species from the genus Actinomyces) and Firmicutes (Peptostreptococcaceae bacterium COT030), that had significantly higher estimated mean proportions with moderate compared to no gingivitis when all the teeth had periodontitis. In contrast, there was one OTU belonging to the Proteobacteria (a novel species from the genus Haemophilus) that had significantly higher predicted proportions with moderate compared to no gingivitis when none of the teeth had periodontitis. There were also three OTUs with an interaction between gingivitis and breed size and one with a significant interaction between gingivitis and age (Supplementary Table 1).

Of the 82 OTUs found to have a significant average gingivitis effect, 39 had a significantly higher estimated mean proportion at a gingivitis score of three compared to a score of zero and 35 had a significantly higher proportion at a gingivitis score of zero compared to a score of three (Supplementary table 2, Fig. 4). Of the OTUs associated with moderate gingivitis the majority belonged to the phylum Firmicutes (20 OTUs), followed by Bacteroidetes (5 OTUs), Spirochaetes (5 OTUs) and Actionbacteria (4 OTUs). The remaining five OTUs belonged to the phyla SR1, TM7, Chloroflexi, Proteobacteria and Synergistetes. The most dominant members of the Firmicutes $(>0.3 \%)$ with odd ratios greater than two were; three species from the Peptostreptococcaceae family (COT-019, COT-077 and COT-004) two species from the class Clostridiales (COT-028 and COT-141), Tissierella sp. COT-029, Erysipelotrichaceae bacterium COT-302, Schwartzia sp. COT063 and three novel species from the genera Filifactor, Peptococcus and Roseburia. With respect to the other phyla the most abundant OTUs $(>0.3 \%)$ that were strongly associated with gingivitis (odds ratio $>2$ ) were Porphyromonas sp. COT-239 and a novel Porphyromonas species which belong to the phyla Bacteroidetes, Treponema denticola a member of the Spirochaetes and Actinomyces sp. COT-407 and a novel species belonging to the phylum Actinobacteria.

Of the 35 OTUs that were at a significantly higher mean proportion at a gingivitis score of zero compared to a score of three, these predominantly belonged to two phyla Proteobacteria (16 OTUs) and Bacteroidetes (10 OTUs) with the remaining nine OTUs belonging to the phyla Firmicutes, Fusobacteria, Actinobacteria and SR1. The most abundant OTUs $(>0.3 \%)$ from the phylum Actinobacteria with an odds ratio $>2$ were Pasteurellaceae bacterium COT-080, Neisseria shayeganii, Cardiobacterium sp. COT-177, Xenophilus sp. COT-264, Pasteurella dagmatis, two species of Lautropia (COT-060 and a novel species) and two novel species from the genera Conchiformibius and Moraxella. In the case of the Bacteroidetes the most 


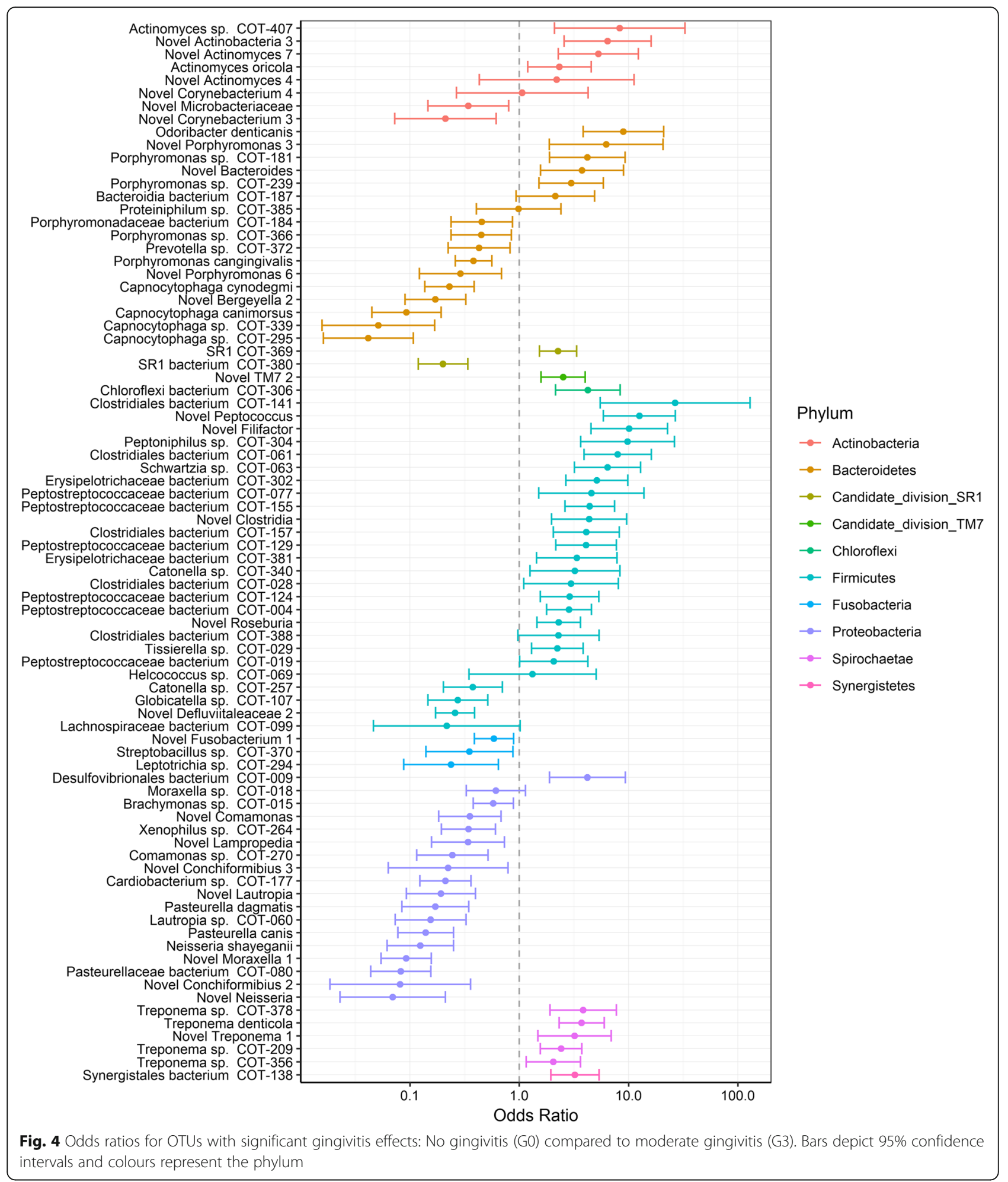

abundant OTUs with an odds ratio $>2$ were four species of Capnocytophaga (C. canimorsus, C. cynodegmi COT-295 and COT-339), Porphyromonas cangingivalis, Porphyromonadaceae bacterium COT-184 and a novel species from the genus Bergeyella.
Of the 33 OTUs found to have a significant percentage healthy teeth effect ( $0 \%$ versus $100 \%$ healthy teeth) only ten were present at relatively high abundance $(>0.3 \%)$ and had an odds ratio $>2$. Four increased with increasing proportion of healthy teeth; a novel species from the 


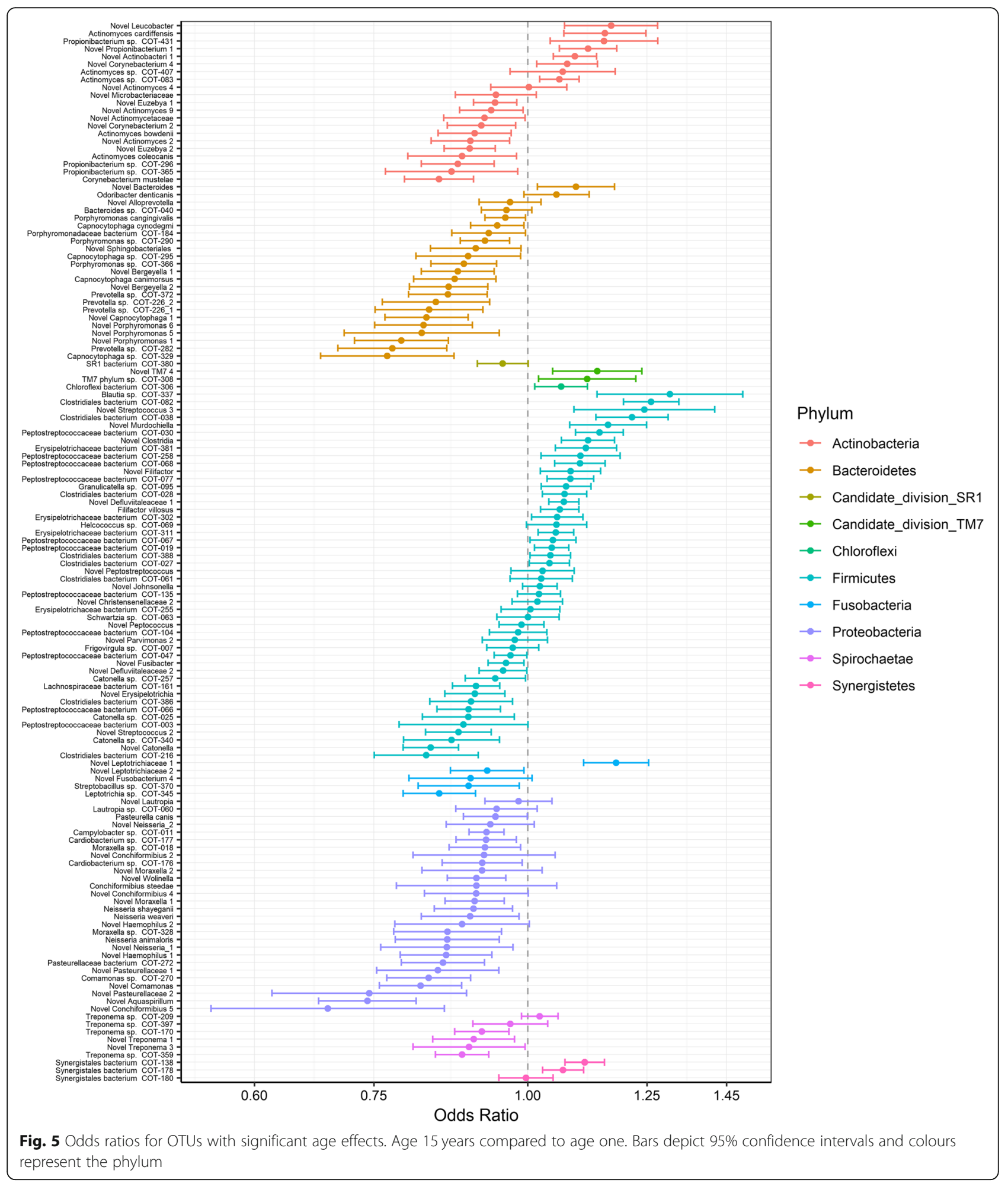

genus Neisseria, Conchiformibius steedae, a novel Peptococcus species and a novel species from the family Pasteurellaceae. Six decreased with increasing proportion of healthy teeth; three species form the Peptostreptococcaceae family, Helococcus sp. COT-069, Actinobacteria bacterium
COT-376 and a novel species from the genus Corynebacterium.

Of the 10 OTUs found to have a significant percentage teeth with periodontitis effect ( $0 \%$ versus $100 \%)$ only three were present at relatively high abundance $(>0.3 \%)$ 
and had an odds ratio $>2$; a novel species from the genus Actinomyces, Treponema sp. COT-356 and Peptostreptococcaceae bacterium COT-030.

\section{Effect of age}

There were seven OTUs that had an interaction between age and clinical status or age and breed size (Supplementary table 1). Of the 138 OTUs without interactions that were found to have significant age effects, 36 had a significantly higher estimated mean proportion at age 15 than age 1 and 70 had a significantly lower proportion at age 15 compared to age 1 . Of those that were significantly more abundant at an older age, the majority belonged to the phyla Firmicutes (22 OTUs) and Actinobacteria (7 OTUs). The remaining seven OTUs were members of the phyla Bacteroidetes, Synergistetes, TM7, Chloroflexi and Fusobacteria (Supplementary table 2, Fig. 5). Within the phylum Firmicutes there were 12 abundant OTUs ( $>0.3 \%$ of population) that had an odds ratio $>2$ when comparing the estimated proportion at age 15 to age one: Four were assigned to the family Peptostreptococcaceae, two to the family Erysipelotrichaceae, two to the class Clostridiales and four could be assigned to the genus or species level (Blautia sp. COT337, Granulicatella sp. COT-095, Filifactor villosus and a novel species belonging to the genus Streptococcus). With respect to members of the phylum Actinobacteria the four most abundant OTUs $(>0.3 \%)$ with an odds ratio greater than two were Actinomyces sp. COT-083, Propionibacterium sp. COT-431 and two novel species one from the genus Corynebacterium and the other from the genus Leucobacter. Of the 70 OTUs that had a significantly lower proportion at age 15 compared to age 1 , the majority belonged to four phyla; Proteobacteria (20 OTUs), Bacteroidetes (19 OTUs), Firmicutes (13 OTUs) and Actinobacteria (11 OTUs). The remaining 11 OTUs belonged to the phyla Fusobacteria and Spirochaetes. With respect to the Proteobacteria phylum the most abundant members $(>0.3 \%)$ with the biggest difference between ages 15 and one (odds ratio $>2$ ) were three species of Neisseria ( $N$. animolaris, $N$. shayeganii, N. weaveri), two species from the genus Moraxella (Moraxella sp. COT-018 and a novel species), two novel species from the family Pasteurellaceae, Campylobacter sp. COT-011 and a novel species from the genus Aquaspirillum. Representing the phylum Bacteroidetes there were two species from the genus Capnocytophaga (C. canimorsus, C. cynodegmi), a novel species from the genus Bergeyella, Prevotella sp. COT-226, Porphyromonadaceae bacterium COT-184 and two species from the genus Porphyromonas (COT-290 and a novel species). In the phylum Firmicutes there were two novel species, one from the genus Catonella and one from the genus Streptococcus. With respect to the phylum Actinobacteria there were two species from the genus Corynebacterium (C. mustelae and a novel species), two novel species from the genus Euzebya, a novel Actinomyces species and Propionibacterium sp. COT-296.

\section{Effect of breed size}

There were eleven OTUs that had an interaction with breed size, three with gingivitis, four with the percentage of periodontitis teeth, two with the percentage of healthy teeth and two with age (Supplementary table 1). Of the 67 OTUs found to have a significant breed size effect, the majority of these $(\sim 90 \%)$ were due to small breeds significantly differing to medium and large breeds (Supplementary table 2). Small breeds of dog generally had higher estimated mean proportions of OTUs from the phylum Actinobacteria and lower estimated proportions of OTUs from the phyla Bacteroidetes and Proteobacteria than medium and large dogs. The phylum Firmicutes was present at higher and lower estimated proportions in small breed dogs compared to medium and large breeds. Despite the gross phylum level differences only six OTUs were present at relatively high abundance ( $>0.3 \%$ of total population) and had an odds ratio $>2$.

\section{Species diversity}

Linear regression analysis of the Shannon diversity indices found significant interactions of geographical location with percentage of periodontitis teeth $(p=0.0003)$. The percentage of periodontitis teeth with geographical location interaction was due to dogs from Thailand having a significantly higher mean Shannon diversity index at $0 \%$ periodontitis teeth but lower indices at $100 \%$ periodontitis teeth than dogs from the other geographical locations $(p=0.003$; Fig. 6a). The mean Shannon diversity index was $4.07(3.93,4.20)$ when none of the sampled teeth had periodontitis reducing to 3.13 $(2.67,3.59)$ when $100 \%$ of the sampled teeth had periodontitis which is a difference of $0.94(0.25,1.62)$. The Shannon diversity index was not significantly correlated with the percentage of periodontitis teeth with respect to dogs from UK, USA and China $(p=0.266, p=0.288$ and $p=0.976$ respectively).

Linear regression analysis of the Shannon diversity indices also identified significant interactions of age with percentage healthy teeth $(p=0.038)$. The Shannon diversity index decreased with age and the difference in means between ages one and 15 increased with increasing percentage of healthy teeth (Fig. 6b): When no healthy teeth were sampled the difference in means was $0.02(-0.24,0.29)$ whereas when all the sampled teeth were healthy the difference was $0.61(-0.06,1.28)$. However, none of these differences in means were significant 

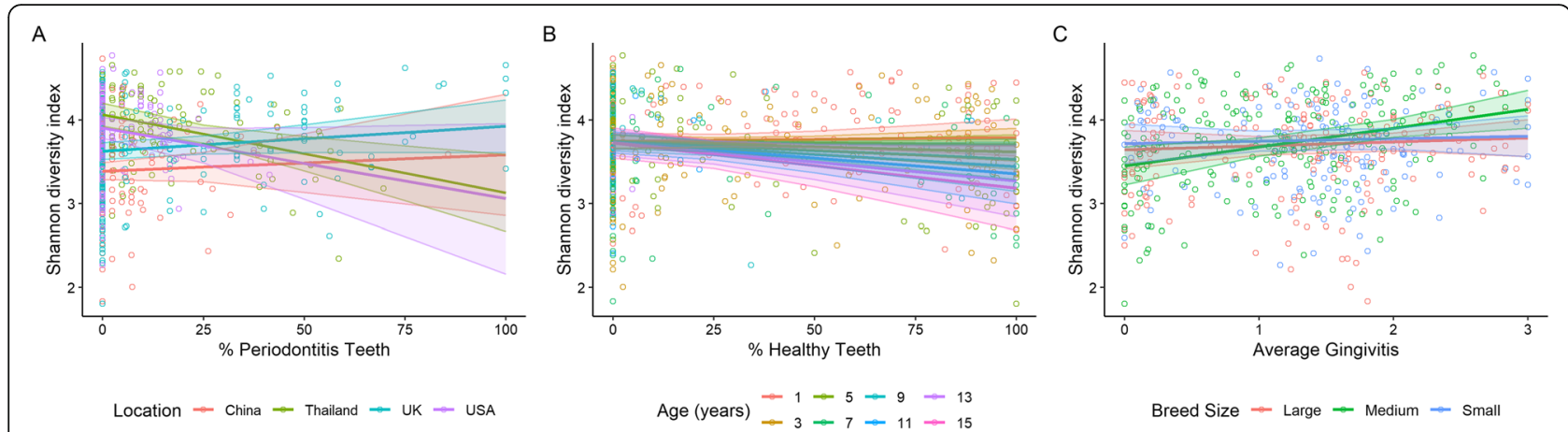

Fig. 6 Shannon diversity indices by A) percentage of teeth with periodontitis and geographical location with raw data represented by open circles coloured by geographical location, B) percentage healthy teeth and age in years with raw data shown as open circles coloured by age and C) average gingivitis score and breed size with raw data represented as open circles coloured by breed size. The average relationships are shown as solid lines with shaded 95\% confidence intervals

$p=0.949$ for $0 \%$ healthy teeth and $p=0.084$ for $100 \%$ healthy teeth.

Linear regression analysis of the Shannon diversity indices also found significant interactions of average gingivitis score with breed size $(p=0.005)$. The average gingivitis score with breed size interaction was due to medium dogs having a significantly lower mean Shannon diversity index at a gingivitis score of zero but higher indices on average at a gingivitis score of $3(p=0.005$; Fig. 6c). The mean Shannon diversity index was 3.45 $(3.22,3.68)$ at a gingivitis score of zero but increased to $4.13(3.9,4.35)$ at a gingivitis score of three which is a difference of $-0.68(-1.18,-0.17)$. There was no correlation between Shannon diversity index and gingivitis score for small and large breed dogs $(p=0.949$ and $p=0.832$ respectively).

\section{Discussion}

This is the largest study to date of the canine oral microbiota; it describes the bacterial composition of subgingival plaque samples from 587 dogs residing in four countries (UK, USA, China and Thailand), determined by 454pyrosequencing. A major strength of the study is that the analysis of all plaque samples was performed in the same laboratory, eliminating the possibility of technical differences in microbial enumeration. A potential weakness however, is that although dogs were selected using the same inclusion and exclusion criteria there were differences in age, breed, breed size and clinical status across countries. This was unavoidable as it was not possible to control for the breeds, sizes and ages of dogs presenting at the veterinary hospitals. To minimise any ethical impact of the study no animals were given a general anaesthesia solely for the purpose of this trial. This was a stipulation of the ethical review body. This consideration did mean that to recruit significant numbers of animals in a reasonable time frame the breed, breed size and age factors were variable. The imbalance in groups was, therefore, unfortunately unavoidable. Breed size and age were therefore included in the statistical models to account for these factors alongside geographical location and periodontal status effects. This also accounted for the fact that periodontal health status, breeds size and age are potentially not independent variables. This ensured that any significant effect on the abundance of an OTU with periodontal health state, for example, was irrespective of the other confounding factor. Likewise, it also enabled the identification of interactions between variables. Another limitation was that plaque sampling methods were different between the UK and the other three geographical locations. In addition, scoring and sampling was undertaken by different individuals at the various geographical locations which could have led to bias. However, all scorers received training by the same Diplomate in Veterinary Dentistry and no discrete clustering of samples by geographical location was observed indicating that different sampling protocols had little effect on the overall plaque microbiota composition. Although, intraoral dental radiographs should be taken as part of a comprehensive periodontal assessment this was not always possible as several of the veterinary hospitals did not have this capability due to cost and availability of the equipment within some of the countries. Scoring of periodontal disease was therefore based on clinical attachment loss, on one or more aspects of the tooth, as measured using a periodontal probe. The probing depths were based on the average estimated root length of a small, medium and large breed of dog and therefore may not be accurate for every tooth in the mouth. The stages of periodontal disease defined by the American Veterinary Dental College are merely descriptive and, for this reason, the scoring criteria used in this study were developed in collaboration with a diplomate of the European Veterinary Dental College (EVDC). The criteria defined were verified using dental radiographs from some of the dogs. 
Overall the bacterial composition of subgingival plaque was comparable to that which has been reported previously $[5,8,9,11,24]$. For example, Firmicutes were the most abundant phyla on average across all samples (29.4\%) followed by Bacteroidetes (19.8\%) and Proteobacteria (14.9\%). Furthermore, the species Porphyromonas cangingivalis dominated with an average abundance of $5.7 \%$ across all samples. Exploration of the effect of geographical location, clinical status, age and breed size on the microbiota revealed that the community composition was most influenced by geographical location, age and gingivitis.

There was a striking resemblance between the bacterial associations with age and gingivitis. The composition of plaque from young dogs and those with no gingivitis was dominated by taxa from the phyla Bacteroidetes and Proteobacteria. Representatives from the phylum Bacteroidetes included species belonging to the genera Capnocytophaga and Bergeyella along with Porphyromonadaceae COT-184. With respect to Proteobacteria species from the genera Neisseria (in particular N. shayeganii), Moraxella and the family Pasteurellaceae were examples of species common to young dogs and those without gingivitis. In contrast, the plaque microbiota of older dogs and those with moderate gingivitis was dominated by members of the Firmicutes. Representatives of this phylum included members of the genus Filifactor, the class Clostridiales (in particular Clostridiales bacterium COT-028) and the families Erysipelotrichaceae and Peptostreptococcaceae. The bacterial associations with gingivitis concur with findings from other studies $[9,11]$. Furthermore, bacterial species belonging to the phylum Firmicutes have been shown to increase in relative abundance as the severity of gingivitis worsens [11]. This is the first study to investigate the effect of age on the canine oral microbiota.

This study showed evidence of a core microbiota in canine subgingival plaque across the UK, USA, China and Thailand; there were three clusters of species which together accounted for about a third of the OTUs and nearly $50 \%$ of the sequences. Furthermore, only about $3 \%$ of the taxa were not shared across geographical locations and the percentage of rare taxa did not significantly differ between countries. However, in terms of relative abundance over $50 \%$ of the OTUs differed across geographical locations and the majority of these were due to differences between the UK and the other three countries. This concurs with human studies in that no significant geographic patterning was observed with respect to the human salivary microbiome but specific genera did vary significantly in frequency among geographical locations [23]. Likewise, DNA-DNA hybridisation studies found that on average all species were detected in subgingival plaque samples from different countries but the proportions of a number of species differed $[15,16]$. This suggests that diet and the environment do not significantly influence the composition of the oral microbiome. However, a study of the saliva microbiome of human groups living under very different climatic conditions showed only limited support for a core microbiome at the OTU level across three continental regions [18].

The most striking observation was that plaque samples from the UK dogs were characterised by higher estimated proportions of species from the phyla Bacteroidetes and Proteobacteria compared to the USA, China and Thailand. The phyla Proteobacteria and Bacteroidetes are associated with young dogs and healthy gingiva suggesting that the UK dogs had microbial profiles similar to young dogs or dogs with low levels of gingivitis compared to other countries. The UK was also depicted by lower proportions of taxa belonging to the phylum Actinobacteria compared to the other three geographical locations and this phylum was also associated with young dogs and dogs with no gingivitis. This finding might be explained by differences in how plaque samples were collected in the UK where a subset of teeth were sampled compared to whole mouth collections in the other three geographical locations. Alternatively, it could be the result of genetics or other environmental factors such as feeding practices and oral care regimes that may exist. Another noticeable trend was that the dogs from China had lower estimated proportions of the phylum Bacteroidetes compared to the UK, USA and Thailand and lower estimated proportions of the phylum Proteobacteria than the UK and Thailand. This might be due to the fact that dogs from China had higher levels of gingivitis compared to Thailand and the USA and a lower percentage of healthy teeth than the other three geographical locations. Bacteroidetes and Proteobacteria were more represented in Thailand than China possibly because the dog population from Thailand was significantly younger by about 2 years. However, dogs from Thailand had a significantly higher proportion of teeth with periodontitis than the other three locations. Despite these differences only six OTUs were shown to have geographical location interactions with clinical status suggesting that bacterial associations with health and disease did not significantly differ by geographical location. Several studies of human subjects have shown that the mean proportions of plaque-associated species differ by country [12-14].

Approximately $25 \%$ of the OTUs were found to have a significant breed size effect and the majority of these were due to small breeds significantly differing to medium and large breeds. This study highlighted that small dogs had higher levels of many Firmicutes species than medium and large breeds, such as members of the family Erysipelotrichaceae and the class Clostridiales, which were also associated with moderate gingivitis. 
Furthermore they had significantly lower predicted proportions of many of the species belonging to the phylum Proteobacteria which were associated with healthy gingiva in this study. This is the first study to explore breed size differences in the oral microbiota and therefore, to the best of our knowledge, there are no published studies to which this data can be compared. It was not possible within this study to investigate the impact of breed due to over 90 breeds within the study cohort and hence low numbers of representatives for each breed. The link between the oral microbiota and breed size might explain why periodontitis is more prevalent in small breeds of dog compared to larger breeds $[2,3,25]$. These differences with respect to breed size might also explain some of the geographical location differences observed as the UK dog population had larger breed dogs, Thailand had more medium sized dogs and the USA had more small dogs than China and Thailand. Studies of ethnic groups in the USA reported differences in the prevalence of microorganisms associated with human periodontitis between whites, Hispanics, and Asian-Americans [26], between African-Americans, Native Americans and Asians [27] and between African-American, Asian-American and Hispanics [17]. Furthermore, a recent study showed evidence of ethnicity-specific clustering of microbial communities in saliva and subgingival biofilms which appeared to be capable of discriminating between ethnicities [19]. Further investigations are required to determine if there is a breed-specific microbial signature.

\section{Conclusions}

We have shown that subgingival plaque from dog populations located in the UK, USA, China and Thailand has a similar bacterial composition although the abundance of certain taxa significantly varies among geographical locations. These differences might be explained by genetics, diet, veterinary practices and oral hygiene procedures. Most important however, is that the bacterial associations with health, gingivitis and periodontitis did not significantly differ by geographical location. This means that it is plausible that the bacterial species within dental plaque can be used to classify dogs with periodontal disease, irrespective of where they reside, resulting in potential improvements in disease detection.

\section{Methods}

\section{Sample population}

The study cohorts comprised client-owned dogs presented at pet hospitals in the UK, USA, China and Thailand. With respect to the UK dog population the bacterial composition of their subgingival microbiota has been described previously [9]. The samples from the
USA were collected from dogs visiting Banfield Pet Hospital in Portland between September 2012 and May 2013. The samples from China were collected from dogs visiting the Meillian Animal hospital in Beijing between March 2013 and July 2014. The samples from Thailand were collected from dogs visiting pet hospitals within Kasetsart University in Huahin and Bangkok between March 2013 and June 2014. An owner survey was completed for all dogs which included questions about the dog's breed, age, sex, neuter status and breed size (small < $10 \mathrm{~kg}$, medium 10-25 kg, large $>25 \mathrm{~kg}$ ).

Purebred and crossbred dogs over 1 year of age were included in the study if they had not received corticosteroids, antibiotics or professional dental cleaning in the preceding 3 months. Dog breeds predisposed to developing periodontitis (Greyhounds, Yorkshire terrier, Maltese and toy/miniature poodles) $[2,3,28]$ were excluded as this may be a different form of the disease. Dogs that had moderate or severe periodontitis ( $>25 \%$ attachment loss [29]) were also excluded. This was defined as two indicator teeth (maxillary 3rd incisor, canine, 3rd and 4th premolar and mandibular canine, 4th premolar and 1st molar) or six teeth in the whole mouth at PD3 or above. Only dogs under general anaesthesia for routine treatment for non-periodontal complications were screened for inclusion in the study. No dogs were anaesthetised solely for the collection of plaque samples.

Prior to the start of the study a sample size calculation was performed using data from our previous UK crosssectional survey [9]. The calculation assumed that the species diversity and variability in the relative abundance of bacterial species in the other three countries would be similar to that observed in the UK dog population. Based on the power calculation a sample size of 35 dogs per health state (health, gingivitis and mild periodontitis) was targeted. This would enable at least a 2 -fold change in relative abundance between health states to be detected for bacterial species present at high abundance ( $>2.68 \%$ of the total population), a 3-fold change for bacterial species present at medium abundance $(>0.37 \%$ of total population) and a 5-fold change for bacterial species present at low abundance $(>0.06 \%$ of total population) with a power of at least $80 \%$ using an overall significance test level of $5 \%$ that incorporates adjustments for multiple testing [30].

\section{Clinical assessment}

Clinical assessments were performed by three to five veterinary nurses or veterinarians at each of the four collection sites. All received a minimum of 2 days training on scoring and recording periodontal disease from a Diplomate of the European Veterinary Dental College (Milella L). 
The extent of gingivitis and periodontitis was assessed by taking measurements at the gingival margin using a periodontal probe. A gingivitis score between 0 and 4 was recorded for every tooth using a modified combination of the gingival index and sulcus bleeding index [29] (Table 4). Probing depth, gingival recession and furcation exposure were recorded according to the criteria in Table 5. Probing depth was measured from the gingival margin to the bottom of the periodontal pocket. Gingival recession was measured from the cementoenamel junction to the gingival margin.

Both were measured using the graduations of a periodontal probe. Total attachment loss was calculated as the sum of the gingival recession and the periodontal probing depth in accordance with established protocols $[31,32]$. PD2 was classified as being up to $25 \%$ attachment loss and periodontitis stage 3 (PD3) as between 25 and $50 \%$ attachment loss, as defined by the American Veterinary Dental College. A dental chart was completed for each dog where, in addition to recording clinical status of each individual tooth as described above, missing teeth, crown fractures below the gingival margin and foreign bodies were documented. Dental radiographs were taken of some of the dogs and were inspected by a Diplomate of the EVDC to confirm the measures used in this study were indicative of early bone loss.

\section{Sample collection}

Subgingival plaque samples were collected at the time of clinical assessment. A sterile periodontal probe was gently inserted under the gingival margin and swept along the base of the crown. For samples collected in the USA, China and Thailand plaque was collected from all the teeth in the mouth and placed into a single Eppendorf tube containing $300 \mu \mathrm{l}$ TE buffer $(10 \mathrm{mM}$ Tris-buffer, 1 $\mathrm{mM}$ EDTA, $\mathrm{pH} 8$ ). If the tooth being sampled was PD3 or there was something that was of concern, for example a foreign body or an oral mass, then the sample was not collected from that tooth. In this instance, to prevent potential contamination of the sample, the probe was changed prior to collecting from other teeth and the exceptions recorded.

To limit the potential bias based on whole-mouth periodontal status, the samples were classified as health, gingivitis or periodontitis. Health was defined as less than two indicator teeth, or less than six teeth in total, with gingivitis. Gingivitis was defined as $\geq 2$ indicator teeth, or six teeth in total, with gingivitis. Early periodontitis was defined as $\geq 3$ indicator teeth, or $\geq 6$ teeth in total, classified as PD2.

The samples were frozen at $-20^{\circ} \mathrm{C}$ within $10 \mathrm{~min}$ of collection. In the USA only, samples were stored for a maximum of $6 \mathrm{~h}$ at $-20^{\circ} \mathrm{C}$ and then transported to a central $-80^{\circ} \mathrm{C}$ facility. This type of facility was not available at the other three collections sites and therefore samples were stored at $-20{ }^{\circ} \mathrm{C}$ until transported to the laboratory on dry ice for microbial analysis.

For the UK, samples were collected slightly differently [9]. For a mouth scored as health or gingivitis, 18 teeth in total were sampled (upper 03-08, lower 04, 08, 09). For a mouth scored as PD2, 6 to 12 teeth in total were sampled (upper 03, 04, 08, lower 04, 08, 09). For seven of the UK samples the incisors were not sampled and in some instances, if teeth were missing or there was a fracture below the gingival margin, alternative teeth were sampled to ensure sufficient sample quantity; these exceptions were recorded. Fourteen of the samples from the original cross-sectional study were excluded from this study due to the fact that the dental charts for the dogs could not be located and therefore the health status of each individual tooth was not known. A summary of the samples utilised in this study is provided in Table 6.

\section{Microbiologic analysis}

DNA was extracted from the plaque samples and the $16 \mathrm{~S}$ rRNA gene amplified according to the method described by Davis et al. [9]. PCR reactions were purified, quantified and multiplexed 454-pyrosequencing libraries created by pooling PCR amplicons in equimolar amounts. Sequences were generated using the GS FLX Titanium series 454 DNA pyrosequencer (454 Life Sciences). All pre-preparation and sequencing was performed by Eurofins Genomics (Ebersberg, Germany). Uni-directional sequencing was initiated from adapter B on the reverse primers. A sequencing depth of 15,000 sequences per sample was targeted which was comparable to that used for the UK cross-sectional study [9].

\section{Sequence processing}

The standard flowgram files (SFF) were initially filtered by selecting reads with at least 360 flows and truncating long reads to 720 flows. Reads were filtered and denoised using the AmpliconNoise software (version V1.21 [33, 34]). For the initial filtering step, reads were truncated when flow signals dropped below 0.7, indicative of poor quality. Subsequently, reads were denoised in three stages; 1) Pyronoise to remove noise from flowgrams resulting from 454 sequencing errors (PyronoiseM parameters -s 60, -c 0.01), 2) Seqnoise to remove errors resulting from PCR amplification (SeqNoiseM parameters $-\mathrm{s} 25,-\mathrm{c} 0.08), 3$ ) Perseus to detect and remove chimeras resulting from PCR recombination. The denoised sequences were then clustered using QIIME v1.7.0. The QIIME script pick_otus.py, which utilises the Uclust v1.2.22q software program, was used to cluster sequences with $\geq 98 \%$ identity [35]. Uclust was run with modified parameters, with gap opening penalty set to 2.0 and gap extension penalty set to 1.0 and $-\mathrm{A}$ 
flag to ensure optimum alignment [35]. Representative sequences of all observed OTUs were annotated using BLAST [36] against the Silva SSU database release 119 [37]. If the alignment matched the top BLAST hit with $\geq 98 \%$ sequence identity and $\geq 98 \%$ sequence coverage then a species level was assigned but if these criteria were not met the next appropriate level of taxonomic assignment was allocated: $\geq 94 \%$ genus; $\geq 92 \%$ family; $\geq 90 \%$ order, $\geq 85 \%$ class, $\geq 80 \%$ phyla.

\section{Phylogenetic tree construction}

OTU representative sequences were blasted against Silva SSU database release 119 , best hit full length $16 \mathrm{~S}$ sequences were used to represent OTUs within the tree. An initial multiple sequence alignment (msa) was constructed using pynast v0.1 [38] and Greengenes core template, this alignment was filtered using filter_alignment.py script from QIIME v1.8.0 [35] with default settings. The filtered msa was realigned using MUSCLE v3.8.31 [39] with default settings and a phylogenetic tree inferred using FastTree v2.1.7 [40] using a generalised time-reversible model. The tree figure was plotted using GraPhlAn v0.9.7 (unpublished).

\section{Statistical analysis}

An OTU was classed as rare if none of the geographical locations had an average proportion above $0.05 \%$ and/or had presence in less than two samples. The $0.05 \%$ cutoff was selected based on statistical analysis of data from mock communities [9].

\section{Baseline analysis}

Total sequence depth, the age (years) and average gingivitis score were analysed by generalised least squares linear models with a fixed effect of geographical location and weighting the variance by geographical location. Means were compared using Tukey HSD tests to the 5\% level and reported with 95\% family-wise confidence intervals. The percentage of rare sequences, healthy teeth and periodontitis teeth were analysed by logistic regression analyses (GLM with a quasi-binomial distribution and logit link) for proportions, using the count of rare sequences out of the total sequence depth. Geographical location was investigated as a fixed effect and means were compared using Tukey HSD tests to the 5\% level. Contingency tables of breed size, sex and neuter status by geographical location were analysed using Chi-square tests for independence using a test level of $5 \%$.

\section{Univariate analysis}

The 280 individual OTUs were analysed univariately by logistic regression analyses (GLM with a quasibinomial distribution and logit link) for proportions, using the count for the OTU out of the total number of sequences. To enable model convergence when an OTU has many zero counts, 2 counts were added to each OTU count and 4 counts were added to the total count (analogous to adding 2 successes and 2 failures [41]) prior to analyses. The models were explored to investigate the correlation of the OTUs with the fixed effects for oral health status, as measured by the percentage of healthy teeth, percentage of periodontitis teeth and the average gingivitis score, and their two way interactions with geographical location and each other. Age and breed size were also explored as covariates. Models were built initially to minimise the quasi - Akaike's Information Criterion (qAIC) for binomial distributions with over dispersion [42], with geographical location fixed to remain in the model. The model with the smallest qAIC were then chosen to be tested for significance. To adjust for multiplicity effect, the $p$-values of each fixed effect in the minimum qAIC model were adjusted according to the false discovery method of Benjamini and Hochberg [30] for

Table 4 Summary of metadata for each of the four locations

\begin{tabular}{|c|c|c|c|c|}
\hline & UK & USA & China & Thailand \\
\hline \multicolumn{5}{|l|}{ Age (years) } \\
\hline Average & 5.78 & 5.61 & 5.02 & 2.67 \\
\hline$(95 \% \mathrm{Cl})$ & $(5.26,6.3)^{b}$ & $(4.93,6.29)^{b}$ & $(4.17,5.87)^{b}$ & $(2.16,3.18)^{a}$ \\
\hline Range & 1.5 to 15 & 1 to 14.5 & 1 to 14 & 0.8 to 14 \\
\hline \multicolumn{5}{|l|}{ Breed size } \\
\hline Small $(<10 \mathrm{~kg})$ & $36(17.6 \%)$ & $56(46.3 \%)$ & $57(42.5 \%)$ & $22(17.7 \%)$ \\
\hline Medium (10-25 kg) & 76 (36.9\%) & $24(19.8 \%)$ & $43(32.1 \%)$ & 81 (65.3\%) \\
\hline Large (> 25 kg) & 93 (45.1\%) & 41 (33.9\%) & $33(24.8 \%)$ & 21 (16.9\%) \\
\hline \multicolumn{5}{|l|}{ Sex } \\
\hline Female & $94(45.2 \%)$ & $54(44.6 \%)$ & 55 (45.1\%) & $59(47.6 \%)$ \\
\hline Male & $114(54.5 \%)$ & $67(54.5 \%)$ & $67(54.5 \%)$ & 65 (52.4\%) \\
\hline
\end{tabular}

\footnotetext{
a, bSignificance with Tukey HSD homogenous groups at $5 \%$ within model
} 
Table 5 Periodontitis scoring criteria

\begin{tabular}{|c|c|c|c|}
\hline AVDC Stage $^{a}$ & $\begin{array}{l}\text { Periodontal Probing } \\
\text { Depth }\end{array}$ & Gingival Recession & Furcation Exposure \\
\hline \multirow[t]{3}{*}{$\begin{array}{l}\text { Stage } 2 \text { (PD2) Early periodontitis }(<25 \% \\
\text { attachment loss) }\end{array}$} & $\begin{array}{l}\text { Large dog } \geq 3 \text { ( } \geq 6 \text { on } \\
\text { canine teeth) }\end{array}$ & $>0$ & $\begin{array}{l}\text { Grade } 1 \text {; feel an indentation between the roots and } \\
\text { the probe may advance } 1 \mathrm{~mm} \text {. }\end{array}$ \\
\hline & $\begin{array}{l}\text { Medium dog } \geq 3 \text { ( } \geq 3 \\
\text { on canine teeth) }\end{array}$ & & \\
\hline & $\begin{array}{l}\text { Small dog } \geq 2 \text { ( } \geq 3 \text { on } \\
\text { canine teeth) }\end{array}$ & & \\
\hline \multirow[t]{3}{*}{$\begin{array}{l}\text { Stage } 3 \text { (PD3) Moderate periodontitis } \\
\text { (25-50\% attachment loss) }\end{array}$} & $\begin{array}{l}\text { Large dog }>5 \text { (>9 on } \\
\text { canine teeth) }\end{array}$ & Large dog $>5 \mathrm{~mm}$ & $\begin{array}{l}\text { Grade 2; obvious indentation between the roots and } \\
\text { probe advances } 50 \% \text {. }\end{array}$ \\
\hline & $\begin{array}{l}\text { Medium dog }>4(>5 \\
\text { on canine teeth) }\end{array}$ & $\begin{array}{l}\text { Medium \& small dog }>3 \\
\mathrm{~mm}\end{array}$ & \\
\hline & $\begin{array}{l}\text { Small dog }>4(>4 \\
\text { on canine teeth) }\end{array}$ & & \\
\hline
\end{tabular}

Large, medium and small dogs were defined as $>25 \mathrm{~kg}, 10-25 \mathrm{~kg}$ and $<10 \mathrm{~kg}$ respectively

ahttps://avdc.org/avdc-nomenclature/

the 280 OTUs analysed. Subsequently effects were removed from the model if found to be nonsignificant by Benjamini and Hochberg with a false discovery rate of $5 \%$ level. Once this minimal model by qAIC and Benjamini and Hochberg adjustment was formed, the data were subjected to a permutation test to assess the sensitivity of the results to possible outliers or deviations from the assumption of the generalised linear model. An effect remained in the model if the proportion of permutations where the significance of the effect was as least as small as the observed effect was less than 5\%. Means and odds ratios between levels were then calculated at the covariate averages according to final model found, with $95 \%$ family wise confidence intervals. $P$-values for comparisons between geographical locations and breed sizes are calculated using a family-wise error rate of $5 \%$.

\section{Shannon diversity}

The Shannon Diversity index of each sample was calculated [43] and analysed by linear regression modelling. Models were built using stepwise regression to minimise the AIC, with fixed effects as defined for the univariate analyses and the total number of sequences and geographical location fixed in the models. The data were then subjected to a permutation test according to the

Table 6 Subgingival plaque samples

\begin{tabular}{llll}
\hline Location & Number of samples & Teeth sampled & Storage conditions \\
\hline UK & 209 & Subset of teeth & $-20^{\circ} \mathrm{C}$ \\
USA & 121 & Whole mouth & $-20^{\circ} \mathrm{C}$ then $-80^{\circ} \mathrm{C}$ \\
China & 133 & Whole mouth & $-20^{\circ} \mathrm{C}$ \\
Thailand & 124 & Whole mouth & $-20^{\circ} \mathrm{C}$ \\
\hline
\end{tabular}

minimised model, as for univariate analyses. A test level of $5 \%$ was used.

\section{Multivariate analysis}

The $\log _{10}$ (proportions) (after 2 counts were added to each OTU count and 4 counts were added to the total count) for the profile were analysed by PCA. Score plots of the components were investigated for correlations with breed size, age, gender, neuter status, average gingivitis score and the percentage of healthy and periodontitis teeth in the mouth and in the sampled teeth.

Statistical analyses were performed in R v3.2.2 statistical software [44]. PCA analysis was performed using the library vegan, comparisons and confidence intervals were calculated using multcomp and the model AICs were calculated using MuMIn. Graphics were generated using ggplot2 [45].

\section{Supplementary Information}

The online version contains supplementary material available at https://doi. org/10.1186/s12917-020-02660-5.

Additional file 1: Table S1. OTUs that had significant interactions with location and other covariates. A) Age by location, B) average gingivitis by location, C) breed size by location, D) age by average gingivitis, E) age by percentage healthy teeth, F) age by percentage periodontitis teeth, $G$ ) average gingivitis by breed size, $\mathrm{H}$ ) average gingivitis by percentage healthy teeth, I) average gingivitis by percentage periodontitis teeth, J) breed size by percentage healthy teeth, K) breed size by percentage healthy teeth, L) Percentage healthy teeth by percentage periodontitis teeth and M) age by breed size. Tables summarise estimated mean proportions, odds ratios, 95\% confidence intervals and $p$-values.

Additional file 2: Table S2. Table summarises estimated mean proportions, odds ratios, 95\% confidence intervals and $p$-values for OTUs that had significant main effects of A) location, B) age, C) average gingivitis, D) breed size, E) Percentage healthy teeth and F) percentage periodontitis teeth. 
Additional file 3: Figure S1. A) proportion of healthy teeth, B) average gingivitis score and C) proportion of periodontitis teeth in sampled teeth compared to whole mouth coloured by location.

Additional file 4: Figure S2. Heatmap of OTU relative abundance. From left to right; hierarchical cluster of OTU presence/absence using Jaccard distance and average agglomeration, clusters of interest are coloured red (cluster A), blue (cluster B) and green (cluster C). OTU best hit annotations and phylum level classification denoted by coloured blocks. The percentage of all samples containing each OTU (presence defined as $>0.05 \%$ ) is displayed using black to white shaded ribbon with a continuous scale where black would be an OTU present in all samples. The heatmap graphic represents the relative abundance of each OTU in each sample, ordered by sample country of origin, black represents relative abundances greater than $5 \%$ and white $0 \%$

\section{Abbreviations}

OTU: Operational taxonomic unit; PD2: Periodontal disease stage 2; PD3: Periodontal disease stage 3; UK: United Kingdom; USA: United States of America; V1: Variable region 1; V3: Variable region 3; GLM: Generalised linear model; Sp: Species; COT: Canine Oral Taxon; PCR: Polymerase chain reaction; DNA: Deoxyribonucleic acid; qAIC: Akaike's Information Criterion; PCA: Principal components analysis

\section{Acknowledgements}

The authors would like to acknowledge the staff at Banfield Pet Hospital in the USA, Kasetsart University in Thailand and Meillian Animal hospital in China for their dedication in collecting all the plaque samples. We would also like to acknowledge Mike Roebuck for creating an Access database to capture the clinical data, Gaelle Thomas and Robyn Bednall for entering all the dental charts into the database and the laboratory team for processing all the samples. In particular we would like to thank Ashlee Addleman, Nathaniel Spofford, Wang Fang and Pailin Petison for managing the collection of plaque samples within their regions.

\section{Authors' contributions}

CW was involved in the conception, design of the study, acquisition, analysis and interpretation of the data and drafting of the manuscript. AC made substantial contributions to the design, analysis and interpretation of the data. $\mathrm{CO}$ analysed and interpreted the data. SH, LH and LM were involved in the conception and design of the study. All authors read and approved the final manuscript.

\section{Funding}

This work was funded by the WALTHAM Petcare Science Institute (Mars Petcare). The authors employed by WALTHAM were involved in the study design, data collection and analysis, decision to publish, and preparation of the manuscript as defined in section on author's contributions.

\section{Availability of data and materials}

Data supporting the conclusions of this manuscript are available from the corresponding author upon reasonable request.

\section{Ethics approval and consent to participate}

Ethical approval for the study was given by the Waltham Animal Welfare and Ethical Review Body (AWERB). Veterinary hospitals did not have their own ethical review boards and therefore adhered to Waltham AWERB requirements and, veterinarians who were conducting routine veterinary dental treatment followed the principles of Good Veterinary Practice and local regulations. All dogs included in the study were already scheduled to undergo anaesthesia for routine dental treatment. Written client consent was obtained for the collection of plaque samples from their dogs whilst under general anaesthesia for this dental treatment and subsequent use of the samples for research purposes.

\section{Consent for publication}

Not applicable.

\section{Competing interests}

CW, AC, CO, SH and LH were employed by the WALTHAM Petcare Science Institute (Mars Petcare) at the time of this research. Two patents have been filed based on this work.

\section{Author details}

'WALTHAM Petcare Science Institute, Mars Petcare UK, Melton Mowbray, Leicestershire, UK. ${ }^{2}$ The Veterinary Dental Surgery, Byfleet, Surrey, UK.

Received: 8 March 2020 Accepted: 30 October 2020

Published online: 06 January 2021

\section{References}

1. Butković V, Šimpraga M, Šehić M, Stanin D, Sušić V, Capak D, et al. Dental diseases of dogs: a retrospective study of radiological data. Acta Vet Brno. 2001:70(2):203-8.

2. Kyllar M, Witter K. Prevalence of dental disorders in pet dogs. Vet Med. 2005; 50(11):496-505.

3. Hamp SE, Olsson SE, Farsø-Madsen K, Viklands P, Fornell J. A macroscopic and radiological investigation of dental diseases of the dog. Vet Radiol. 1984;25(2):86-92.

4. Williams RC. Periodontal disease. N Engl J Med. 1990;322(6):373-82.

5. Dahlén G, Charalampakis G, Abrahamsson I, Bengtsson L, Falsen E. Predominant bacterial species in subgingival plaque in dogs. J Periodontal Res. 2012;47(3):354-64.

6. Elliott DR, Wilson M, Buckley CMF, Spratt DA. Cultivable oral microbiota of domestic dogs. J Clin Microbiol. 2005:43(11):5470-6.

7. Hardham J, Dreier K, Wong J, Sfintescu C, Evans RT. Pigmented-anaerobic bacteria associated with canine periodontitis. Vet Microbiol. 2005;106(1-2): 119-28

8. Dewhirst FE, Klein EA, Thompson EC, Blanton JM, Chen T, Milella L, et al. The canine oral microbiome. PLoS One. 2012;7(4):e36067.

9. Davis IJ, Wallis C, Deusch O, Colyer A, Milella L, Loman N, et al. A crosssectional survey of bacterial species in plaque from client owned dogs with healthy gingiva, gingivitis or mild periodontitis. PLoS One. 2013;8(12): e83158.

10. Sturgeon A, Stull JW, Costa MC, Weese JS. Metagenomic analysis of the canine oral cavity as revealed by high-throughput pyrosequencing of the 165 rRNA gene. Vet Microbiol. 2013;162(2-4):891-8.

11. Wallis C, Marshall M, Colyer A, O'Flynn C, Deusch O, Harris S. A longitudinal assessment of changes in bacterial community composition associated with the development of periodontal disease in dogs. Vet Microbiol. 2015;181(34):271-82.

12. Sanz M, van Winkelhoff AJ, Herrera D, Dellemijn-Kippuw N, Simon R, Winkel E. Differences in the composition of the subgingival microbiota of two periodontitis populations of different geographical origin. A comparison between Spain and the Netherlands. Eur J Oral Sci. 2000;108(5):383-92.

13. Schenkein HA, Burmeister JA, Koertge TE, Brooks CN, Best AM, Moore LV, et al. The influence of race and gender on periodontal microflora. J Periodontol. 1993:64(4):292-6.

14. Cao CF, Aeppli DM, Liljemark WF, Bloomquist CG, Bandt CL, Wolff LF. Comparison of plaque microflora between Chinese and Caucasian population groups. J Clin Periodontol. 1990;17(2):115-8.

15. Haffajee AD, Bogren A, Hasturk H, Feres M, Lopez NJ, Socransky SS. Subgingival microbiota of chronic periodontitis subjects from different geographic locations. J Clin Periodontol. 2004;31(11):996-1002.

16. Haffajee AD, Japlit M, Bogren A, Kent RL Jr, Goodson JM, Socransky SS. Differences in the subgingival microbiota of Swedish and USA subjects who were periodontally healthy or exhibited minimal periodontal disease. J Clin Periodontol. 2005;32(1):33-9.

17. Craig RG, Boylan R, Yip J, Bamgboye P, Koutsoukos J, Mijares D, et al. Prevalence and risk indicators for destructive periodontal diseases in 3 urban American minority populations. J Clin Periodontol. 2001;28(6):524-35.

18. Li J, Quinque D, Horz HP, Li M, Rzhetskaya M, Raff JA, et al. Comparative analysis of the human saliva microbiome from different climate zones: Alaska, Germany, and Africa. BMC Microbiol. 2014;14:316.

19. Mason MR, Nagaraja HN, Camerlengo T, Joshi V, Kumar PS. Deep sequencing identifies ethnicity-specific bacterial signatures in the oral microbiome. PLoS One. 2013:8(10):e77287.

20. Contreras M, Costello EK, Hidalgo G, Magris M, Knight R, Dominguez-Bello MG. The bacterial microbiota in the oral mucosa of rural Amerindians. Microbiology. 2010;156(Pt 11):3282-7.

21. Nasidze I, Li J, Schroeder R, Creasey JL, Li M, Stoneking M. High diversity of the saliva microbiome in Batwa pygmies. PLoS One. 2011;6:8. 
22. Lafaurie Gl, Contreras A, Baron A, Botero J, Mayorga-Fayad I, Jaramillo A, et al. Demographic, clinical, and microbial aspects of chronic and aggressive periodontitis in Colombia: a multicenter study. J Periodontol. 2007;78(4):629-39.

23. Nasidze I, Li J, Quinque D, Tang K, Stoneking M. Global diversity in the human salivary microbiome. Genome Res. 2009;19(4):636-43.

24. Riggio MP, Lennon A, Taylor DJ, Bennett D. Molecular identification of bacteria associated with canine periodontal disease. Vet Microbiol. 2011; 150(3-4):394-400.

25. Harvey CE, Shofer FS, Laster L. Association of age and body weight with periodontal disease in north American dogs. J Vet Dent. 1994;11(3):94-105.

26. Sirinian G, Shimizu T, Sugar C, Slots J, Chen C. Periodontopathic bacteria in young healthy subjects of different ethnic backgrounds in Los Angeles. J Periodontol. 2002;73(3):283-8.

27. Alpagot T, Wolff LF, Smith QT, Tran SD. Risk indicators for periodontal disease in a racially diverse urban population. J Clin Periodontol. 1996; 23(11):982-8.

28. Hoffman T, Gaengler P. Epidemiology of periodontal disease in poodles. J Small Anim Pract. 1996;37:309-16.

29. Wiggs R, Lobprise H. Periodontology. In: Veterinary dentistry: principles and practice. Volume chapter 8. 1st ed. Philadelphia: Lippencott-Raven; 1997. p. 186-232.

30. Benjamini $Y$, Hochberg Y. Controlling the false discovery rate: a practical and powerful approach to multiple testing. J Royal Stat Soc Series B. 1995; 57:289-300.

31. Gorrel C. Periodontal disease. In: Veterinary dentistry for the general practitioner. 1st ed. Philadelphia: Saunders Ltd; 2004. p. 87-110.

32. Harvey CE. Management of periodontal disease: understanding the options. Vet Clin North Am Small Anim Pract. 2005;35(4):819-36.

33. Quince C, Lanzen A, Davenport RJ, Turnbaugh PJ. Removing noise from pyrosequenced amplicons. BMC Bioinform. 2011;12:38.

34. Quince C, Lanzen A, Curtis TP, Davenport RJ, Hall N, Head IM, et al. Accurate determination of microbial diversity from 454 pyrosequencing data. Nat Methods. 2009;6(9):639-41.

35. Caporaso JG, Kuczynski J, Stombaugh J, Bittinger K, Bushman FD, Costello EK, et al. QIIME allows analysis of high-throughput community sequencing data. Nat Methods. 2010;7(5):335-6.

36. Altschul SF, Madden TL, Schaffer AA, Zhang J, Zhang Z, Miller W, et al. Gapped BLAST and PSI-BLAST: a new generation of protein database search programs. Nucleic Acids Res. 1997;25(17):3389-402.

37. Pruesse E, Quast C, Knittel K, Fuchs BM, Ludwig W, Peplies J, et al. SILVA: a comprehensive online resource for quality checked and aligned ribosomal RNA sequence data compatible with ARB. Nucleic Acids Res. 2007;35(21):7188-96.

38. Caporaso JG, Bittinger K, Bushman FD, DeSantis TZ, Andersen GL, Knight R. PyNAST: a flexible tool for aligning sequences to a template alignment. Bioinformatics. 2010;26(2):266-7.

39. Edgar RC. MUSCLE: a multiple sequence alignment method with reduced time and space complexity. BMC Bioinform. 2004;5:113.

40. Price MN, Dehal PS, Arkin AP. FastTree 2--approximately maximumlikelihood trees for large alignments. PLoS One. 2010;5(3):e9490.

41. Agresti A, Coull AB. Approximate is better than 'exact' for interval estimation of binomial proportions. Am Stat. 1998;52:119-26.

42. Burnham A. Model selection and multimodel inference. A practical information-theoretic approach; 2002. p. 67.

43. Shannon CE. The mathematical theory of communication. MD Comput. 1997;14(4):306-17.

44. R: A language and environment for statistical computing [www.R-project.org].

45. Wickham H. ggplot2: elegant graphics for data analysis: Springer-Verlag New York; 2009

\section{Publisher's Note}

Springer Nature remains neutral with regard to jurisdictional claims in published maps and institutional affiliations.

Ready to submit your research? Choose BMC and benefit from:

- fast, convenient online submission

- thorough peer review by experienced researchers in your field

- rapid publication on acceptance

- support for research data, including large and complex data types

- gold Open Access which fosters wider collaboration and increased citations

- maximum visibility for your research: over $100 \mathrm{M}$ website views per year

At BMC, research is always in progress.

Learn more biomedcentral.com/submissions 